Exactitud de la Técnica Poligráfica Zona de Comparación Federal en un Caso de Hurto Simulado

\author{
Martha Cecilia Beltrán Rincón \\ Diego Mauricio Rodríguez Salguero
}

Directora de trabajo de grado

Ps. Ángela Cristina Tapias Saldaña

Universidad Santo Tomas

Facultad de Psicología

Posgrados en Psicología Jurídica

Maestría en Psicología Jurídica

Bogotá, septiembre 2017 


\section{Exactitud de la Técnica Poligráfica Zona de Comparación Federal en un Caso de Hurto Simulado}

\section{Resumen}

La detección del engaño es una solicitud recurrente para la psicología jurídica, pero en particular para la psicología forense y del testimonio. En Colombia hay un amplio uso de la poligrafía, lo cual motiva al estudio de sus técnicas. Por lo anterior se realizó un estudio de diseño preexperimental utilizando la técnica poligráfica Zona de Comparación Federal (ZCT en adelante) en un hurto simulado con actores. Los participantes fueron 30 sujetos de sexo masculino y femenino, estudiantes universitarios de distintas facultades, con un rango de edad entre los 17 y 31 años. El primer grupo conformado por 10 actores universitarios, se denominó grupo de responsables, el segundo grupo de 10 observadores de la simulación con conocimiento del simulacro, se llamó grupo de conocedores y el último grupo compuesto por 10 sujetos inocentes, se denominó grupo control. Todos ellos fueron sometidos a un examen poligráfico mediante la técnica ZCT, por parte de un psicofisiólogo forense.

Una vez terminada la investigación, se puede concluir que la técnica poligráfica Zona de Comparación Federal, arrojó un porcentaje de exactitud en el análisis de la credibilidad del testimonio en eventos específicos del $76 \%$, lo que resulta un aporte importante para la labor de los psicólogos forenses en casos relacionados con delitos. No obstante es un reto estudiar técnicas poligráficas con mayor validez.

Palabras clave: poligrafía, exactitud, psicología del testimonio, credibilidad, Zona de Comparación Federal (ZCT), hurto simulado. 


\begin{abstract}
The detection of deception is a recurring request for legal and testimony psychology. In Colombia there is an extensive use of polygraph, which motivates the study of its techniques. Because of the above a preexperimental design study of the technical Federal ZCT comparison (ZCT onwards) was performed in a simulated theft with actors. Subjects participants were 30 sex male and female, university students from different faculties, with ages ranging from 17 to 31 years old. The first group comprised of 10 university actors, was called responsible, the second group of 10 observers of the simulation with knowledge of the simulacrum was called connoisseurs and the last group consisting of 10 innocent subjects was called control group. All of them were subjected to polygraph examination with technical ZCT and helped by an examiner who was unaware of the role of each of the evaluated.
\end{abstract}

At the end of the research process, it can be concluded that polygraphic technique Federal Comparison Zone throw a percentage of accuracy in the analysis of the credibility of the testimony in specific events of $76 \%$, which is an important contribution in the work for the forensic psychologists in cases related to crimes. However it's a challenge study polygraphic techniques with greater value.

Keywords: polygraph, accuracy, psychology of testimony, credibility, Federal Zone Comparison Technique (ZCT), simulated theft. 


\section{Introducción}

Una de las principales áreas de la psicología jurídica es la psicología del testimonio, siendo esta a su vez una aplicación de la psicología experimental, justo en el punto de convergencia con el derecho, en el cual el psicofisiólogo forense o poligrafista puede intervenir de manera estratégica emitiendo un concepto en torno a la credibilidad del testimonio de personas procesadas por delitos, ya que la prueba psicológica de los peritos está incluida en las pruebas científicas que aportan los hallazgos y conocimientos provenientes de la psicología en el ejercicio del derecho penal y su función juzgadora (Manzanero \& Muñoz, 2011; Manzanero, 2010).

Asimismo, debido a la extensión de las técnicas de la psicología experimental a diferentes ámbitos de aplicación, como la psicología jurídica, los diversos instrumentos o herramientas psicofisiológicas utilizadas para la evaluación de la credibilidad del testimonio, en particular el polígrafo, pone de manifiesto el uso adecuado que se le debe dar, ya que su función es determinar si el testimonio de un sujeto tiene credibilidad con base en el registro de sus reacciones psicofisiológicas ante el temor de ser descubierto a preguntas a las cuales posiblemente vaya a mentir.

Ahora bien, la técnica poligráfica ZCT busca evaluar un testimonio, sin la pretensión de erigirse como instrumento definitivo para detectar mentirosos. El psicofisiólogo forense establece si hay o no engaño con base en respuestas fisiológicas de los interrogados, pero la determinación de la credibilidad o de inocencia o culpabilidad es tarea exclusiva de los jueces y del sistema judicial, por lo que el resultado del examen poligráfico no será determinante ni el único concepto válido, sino que se podrá constituir como uno de los elementos del material probatorio en una investigación judicial. 
Mediante el análisis de las respuestas psicofisiológicas de los participantes en torno a la simulación de un hurto, se pretende evaluar la exactitud de la técnica poligráfica ZCT en el ámbito de la psicología del testimonio.

En el presente estudio se plantea una investigación mediante un hurto simulado cuyo escenario es la Universidad Santo Tomas; la pregunta que orienta la formulación del problema y el objetivo de esta investigación es: ¿qué tan exacta es la técnica poligráfica Zona de Comparación Federal (ZCT) en un caso de hurto simulado? La hipótesis de trabajo sugiere que existe una relación entre dos variables, a saber: el resultado poligráfico y el tipo de participación en el hurto, relación a partir de la cual es posible determinar la exactitud de la ZCT.

El objetivo general del estudio, es evaluar la exactitud de la técnica poligráfica Zona de Comparación Federal en un caso de un hurto simulado entre los grupos participantes del experimento, en la Universidad Santo Tomás. Adicionalmente se propone identificar el efecto de la participación de los distintos grupos de la situación experimental en la variable dependiente con el uso de la Zona de Comparación Federal (ZCT) en la situación de hurto simulado. 


\section{Psicología del testimonio y poligrafía}

Las investigaciones y la literatura existente sobre el análisis de la credibilidad de los relatos y testimonios en el contexto penal se dividen en tres líneas: primero, el análisis del contenido del testimonio, que se enfoca en sus dimensiones semánticas y es la línea más consolidada de la actualidad; segundo, las mediciones psicofisiológicas, que evalúan los cambios fisiológicos del organismo relacionados con la mentira; y tercero, el análisis del comportamiento gestual y no verbal (Ruiz, Llor \& Valverde, 2013).

La psicología del testimonio es una especialidad sumamente compleja cuyo objetivo es estudiar las variables que intervienen en la naturaleza del testimonio, entendido como la información que suministra una persona que ha presenciado directamente un suceso (Quejereta, 1999).

La psicología del testimonio es una subespecialidad de la psicología jurídica por su campo de aplicación dentro de la psicología forense, y tiene como objeto de estudio el testimonio y los factores que median su exactitud, teniendo en consideración la forma como los procesos superiores, en especial la memoria, la percepción y la atención influyen en el mismo. (Espinosa, 2011, p.198).

Asimismo, la psicología del testimonio contempla la credibilidad y la exactitud como sus dos principales dimensiones de análisis. La primera dimensión se refiere al origen de la información proveniente de los testigos (sea esta falsa, cierta o imaginaria) y su interpretación se ha hecho desde la psicología cognitiva y áreas afines como el lenguaje y la comunicación. La segunda dimensión se relaciona con variables de memoria, percepción y atención, que influyen en la precisión de los testimonios de los testigos presenciales (Manzanero, 2010). 
Una de las líneas dentro del estudio de la credibilidad del testimonio, como se mencionó anteriormente, es la relacionada con las reacciones psicofisiológicas de los sujetos. Para tal efecto, el principal método que es empleado para el registro y evaluación de las reacciones de un individuo es la poligrafía.

La poligrafía es una disciplina utilizada como una herramienta para evaluar los registros psicofisiológicos que pueden determinar la credibilidad del testimonio de un individuo; los registros que realiza el polígrafo de las reacciones psicofisiológicas, dependen principalmente de la activación del sistema nervioso autónomo (SNA) cuando un sujeto teme ser descubierto en el engaño. El SNA es la parte del sistema nervioso encargado de conservar la homeostasis corporal. Las terminaciones nerviosas se encuentran en el músculo liso (como los presentes en vasos sanguíneos, la pared del tubo digestivo y de la vejiga), miocardio y glándulas (como las sudoríparas y las salivales). Uno de los sensores del polígrafo, el GSR (Galvanic Skin Response) o EDA (ElectroDermic Activity), colocado en los dedos índice y anular de cualquiera de las manos, registra los cambios presentados en las glándulas sudoríparas y la alteración que generan en la electricidad de la piel, convirtiéndose en uno de los canales de registro más importantes para la calificación de las gráficas tomadas durante el examen de polígrafo. El SNA tiene dos divisiones mayores anatómicamente diferentes: los sistemas nerviosos simpático y parasimpático (Barret, Barman, Boitano \& Brooks, 2012). De estas subdivisiones del SNA, depende la activación y el retorno a la homeóstasis de las reacciones psicofisiológicas que registra el polígrafo.

Producto del análisis de estas reacciones del SNA, se pueden emitir los resultados en los exámenes poligráficos, y para los casos específicos como en el presente estudio, los resultados son de tres tipos: NDI que por sus siglas en ingles significa "No Deception Indicated", es decir 
que el sujeto no decepcionó en el examen, esto es, aprobó el polígrafo. Por el contrario, el resultado DI "Deception Indicated”, sugiere que el evaluado no aprobó su examen poligráfico. De forma tal que los resultados de la evaluación, ya sean NDI o DI, dependerán de la activación del sistema simpático ante las preguntas comparativas, en un sujeto presuntamente inocente o ante las preguntas relevantes en un sujeto que esté presentando conducta engañosa, respectivamente. Finalmente, el resultado Inconcluso "INC", se emite cuando los puntaje obtenidos luego de la prueba no permiten emitir un diagnóstico DI o NDI, pues se encuentran dentro los puntajes de corte, que para la ZCT son -3 (DI) y +1 (NDI).

En la actualidad, los instrumentos poligráficos poseen dispositivos de diferentes canales que son capaces de registrar o grabar tres parámetros fisiológicos: el primero de ellos es el cambio en la respiración (expansión del tórax y las características del flujo de aire en la inhalación y la respiración), el segundo es el cambio en la resistencia eléctrica y la conductividad de la piel (por medio de electrodos colocados en las palmas o los dedos de las manos) y, finalmente, el cambio en la presión arterial (Budahazi, 2012).

Con base en este sustento fisiológico, la poligrafía ha encontrado un amplio espectro de aplicación, como lo mencionan Gómez, Farfán \& López (2016), y es el caso del gobierno colombiano que anunció que sometería a prueba de polígrafo a los funcionarios que participan en la elaboración de pliegos de licitaciones o en la adjudicación de contratos en el sector de infraestructura, vivienda y agua, como herramienta para luchar contra la corrupción y para blindar los procesos de contratación. Asimismo, los autores afirman que, en circunstancias particulares se han aplicado, igualmente, pruebas de polígrafo a fiscales o funcionarios del Cuerpo Técnico de Investigación, que intervienen en las pesquisas de delitos relacionados con 
tráfico de drogas, como requisito para acceder a especializaciones o cursos de capacitación en las agencias norteamericanas que apoyan la lucha contra delitos de tráfico.

A nivel internacional el polígrafo en el momento es empleado en 68 países, 16 de América Latina, utilizado en agencias de seguridad, en selección de personal e investigaciones privadas; además es utilizado como prueba judicial, específicamente en Guatemala y Panamá. En Estados Unidos, el polígrafo se admite como prueba judicial mediante un acuerdo entre el fiscal y el defensor. Friedman (citado por Tapias, Avendaño, Fuentes \& Zaldúa, 2003).

Dentro del contexto internacional otros países en los que se utiliza el polígrafo en áreas relacionadas directamente con el derecho y los delitos, se encuentra Hungría. En este país el polígrafo puede realizarse tanto para el acusado como para el testigo; antes del 2011, de acuerdo a su Código de Procedimiento Penal, sólo el acusado podía ser objeto de examen de polígrafo, mientras que luego de este año el Parlamento Húngaro señaló que los testigos también deberían ser evaluados en casos de gran importancia (Budahazi, 2012).

Otro ejemplo del uso del polígrafo es China, país en el cual el empleo de la prueba psicofisiológica ha crecido rápidamente. Esto se debe en gran parte a que el sistema judicial de China es altamente centralizado y sustantivo en su naturaleza, en comparación con los sistemas de Occidente que tienden a ser descentralizados y de naturaleza procesal. En China no existen asociaciones formales de poligrafistas; sin embargo, en julio de 2004, la Asociación de Ciencias Forenses de China (China Forense Science Association), cuyos miembros son empleados como especialistas forenses dentro de los muchos organismos encargados para hacer cumplir la ley, han creado una nueva sección denominada "China Forensic Science Association PsychoInformation-Probe Professional Committee". Este comité tiene aproximadamente 140 
miembros, que incluye practicantes de todos los organismos gubernamentales relevantes, además de las personas empleadas por las empresas que fabrican los polígrafos. (Zhang, 2011).

La aplicación del polígrafo también se observó en un caso icónico en Paquistán, donde se llevó a cabo un examen de polígrafo para determinar la credibilidad en el testimonio de un sospechoso de homicidio en la vida real. Después de la realización de la Técnica de Entrevista de Evaluación Forense (FAINT por sus siglas en inglés) y el examen de polígrafo utilizando la Técnica de Comparación Zona Integrada, en inglés The Integrated Zone Comparison Technique (IZCT), se encontró que el sospechoso registró indicadores positivos de engaño; su puntuación total en FAINT era -6, indicativo de engaño. Los resultados fueron consistentes con las investigaciones de toxicología y patología forense. Después de estas evidencias, el sospechoso confesó el asesinato a la policía (Ayoub, 2016).

Además del uso del polígrafo en exámenes evidenciarios relacionados con la justicia, existen otras aplicaciones de esta técnica de detección de engaño, ya que es cada vez más común su intervención en el área de talento humano de algunas empresas privadas, que utilizan el polígrafo para reconocer la totalidad de las capacidades del personal que se quiere contratar. El uso del polígrafo se advierte como una valiosa herramienta técnica pues permite verificar la información suministrada, y además sirve como elemento de persuasión, es decir, es un motivador disuasivo, ya que si se advierte su uso frecuente el funcionario de entidades e instituciones públicas y privadas asumirán conductas acordes a las funciones que ejercen en los cargos y puesto de trabajo, de igual manera previene la comisión de actos indebidos y conductas reprochables (Bohada, García, Ortíz \& Oses 2011).

El polígrafo se suele utilizar en la realización de exámenes de ingreso y mantenimiento laboral, además de otros casos específicos que, generalmente, hacen parte de la investigación 
relacionada con delitos para establecer la comisión directa o la participación de los examinados en estos hechos (Guerrero, 2013).

La prueba con polígrafo se ha convertido entonces en un método válido y ampliamente utilizado por grandes empresas, especialmente del sector privado, para hacer procesos de selección y contratación de personal más confiables.

El polígrafo también es aplicable en puntos intermedios entre lo organizacional y lo judicial, como por ejemplo Colombia, en donde una de las estrategias más empleadas por los grupos de delincuencia organizada y organizaciones subversivas consiste en infiltrar personas dentro de las organizaciones para extraer información y causar daño desde el interior de la misma, por lo que el uso de las técnicas de poligrafía fue de uso exclusivo para los cuerpos de inteligencia y seguridad del Estado; hoy en día las empresas privadas también hacen uso de estas herramientas en sus procesos de selección de personal (Farfán, 2014), al punto que existe la resolución 2593 de 2003 de la Superintendencia de Vigilancia y Seguridad Privada, que regula la utilización del polígrafo por parte de los servicio de vigilancia y seguridad privada y se admite el polígrafo como método para la selección de personal.

Sin embargo, el polígrafo no ha sido admisible como prueba judicial, según lo comentado por Gómez \& Farfán (2014), cuando citan a la Corte Suprema de Justicia, en Sala de Casación Penal, Proceso 2647 de 01 de agosto de 2008: “el polígrafo es un procedimiento contrario a la dignidad del sujeto debido al proceso dramático de instrumentalización a que se somete a la persona, de quien se extraen mediciones tomadas del monitoreo de las reacciones del sistema nervioso autónomo, para convertir al propio individuo en instrumento de corroboración de una verdad a la que debe llegar la administración de justicia con absoluto respeto por la dignidad humana. En suma, todas esas razones llevan a la Sala a colegir que el polígrafo no es admisible 
como medio de prueba en el contexto de la teleología de la investigación penal". No obstante existen contraargumentos para rechazar las posturas de las Altas Cortes, ya que no se evalúan simples reacciones fisiológicas, sino la reactividad ante respuestas cerebrales conscientes de un sujeto que ha consentido la evaluación y adicionalmente puede considerarse como un instrumento que permite aportar argumentos para que el juez tome una decisión, sin riesgo alguno de reemplazar su decisión judicial. Sumado a esto, dentro de los argumentos esgrimidos por Tapias (2012) en respuesta a las posturas de la Corte, la autora afirma que la detección psicofisiológica del engaño registra unas respuestas del sujeto emanadas por el sistema nervioso autónomo, unas respuestas que la persona no puede controlar, de allí viene su validez, pero durante la prueba poligráfica no sólo intervienen los registros fisiológicos, la persona brinda su aquiescencia con carácter legal (consentimiento), da acceso a su fuero interno para someterse al examen, el cual requiere de una entrevista con profundidad, en la que sí participan las funciones psicológicas superiores, como pensamiento, lenguaje, memoria, y luego de forma voluntaria y con toda su capacidad mental, particularmente la de comprensión, porque el examinado responde a unos cuestionamientos directos sobre los hechos cuyo correlato es la fisiología.

De acuerdo con Gómez \& Farfán (2016), pese al juicio desfavorable de la Corte Suprema de Justicia en torno a la utilización de los resultados de la prueba del polígrafo como elemento probatorio dentro de un proceso judicial, el uso de esta técnica puede defenderse jurídicamente con base en "estándares convencionales de derechos humanos".

Otro argumento a favor del uso del polígrafo es la "libertad de prueba", tal y como lo menciona el Artículo 373 del Código de Procedimiento Penal Colombiano: "los hechos y circunstancias de interés para la solución correcta del caso, se podrán probar por cualquiera de los medios establecidos en este código o por cualquier otro medio técnico o científico, que no 
viole los derechos humanos", así como en un análisis de la ley estatutaria 1621 de 2013 y su Artículo 2 en torno a los procedimientos de inteligencia y contrainteligencia ya que según esta, el único límite para la aplicación de pruebas poligráficas sería el respeto de los derechos fundamentales. En este mismo sentido, dentro de la ley estatutaria, se prevé que la no superación de las pruebas de credibilidad y confiabilidad, incluido el polígrafo, permitirá negar el acceso e inclusive el retiro del mismo, con lo que el resultado del detector de mentiras recibe la categoría o rango de prueba de una justa causa de despido. Lo anterior revela que parte del ordenamiento jurídico nacional considera al polígrafo una prueba o medio de convicción para acreditar hechos jurídicamente relevantes. Esto constituye un argumento de refuerzo que debe conducir necesariamente a su admisibilidad y licitud, también en los procesos penales (Gómez, Farfán, \& López 2016).

Uno de los pocos estudios realizados en el país fue elaborado por Tapias (2012), en el que reseña la experiencia del uso judicial del polígrafo como una de las técnicas de evaluación dentro de la pericia psicológica forense y evidencia la manera en que se pudo introducir la psicofisiología forense al proceso judicial. En esta misma dirección apunta Tapias (2012), quien apela al artículo 382 de la Ley 906 del 2004 del Código de Procedimiento Penal colombiano para defender el uso del polígrafo como un test más de los que se usan dentro del peritaje psicológico e idóneo para ejercer el peritaje técnico dentro de un conjunto de elementos materiales que puedan constituir un "acervo probatorio". En efecto, en tal artículo se establece bajo el concepto de medio de conocimiento como "cualquier [...] medio técnico o científico, que no viole el ordenamiento jurídico" (Articulo382).

Otra de las normas que es posible citar para justificar la inclusión del polígrafo dentro de un proceso judicial, es el artículo 404 de la ley 906 de 2004, el cual indica que 
“para apreciar el testimonio, el juez tendrá en cuenta los principios técnico-científicos sobre la percepción y la memoria y, especialmente, lo relativo a la naturaleza del objeto percibido, al estado de sanidad del sentido o sentidos por los cuales se tuvo la percepción, las circunstancias de lugar, tiempo y modo en que se percibió, los procesos de rememoración, el comportamiento del testigo durante el interrogatorio y el contrainterrogatorio, la forma de sus respuestas y su personalidad"(Artículo 404).

Vilariño, Formosinho \& Jesús (2012) afirman que la la insuficiencia de pruebas exige al juez dictar su sentencia con base en los testimonios de las partes, teniendo que fundarse en elementos subjetivos de los relatos presentes en un gran número de estos casos, de modo que la intervención de un experto en poligrafía, o un psicólogo forense, puede evaluar tanto la precisión como la credibilidad de las declaraciones (p.599)

Con base en las consideraciones anteriores y las condiciones que establece el artículo 422 del Código de Procedimiento Penal Colombiano (título de admisibilidad de publicaciones científicas y de prueba novel), en torno a los requisitos de admisibilidad de una opinión pericial dentro de un juicio, la presente investigación utiliza el polígrafo como herramienta técnica para la adquisición de elementos probatorios sobre la credibilidad de un testimonio, con base en la determinación de la exactitud de la técnica poligráfica ZCT por medio de hurto simulado.

Esta investigación ofrece una perspectiva prácticamente desconocida hasta el momento en cuanto a la realización de estudios con diseño preexperimental por medio de delitos simulados y su relación con la exactitud de las técnicas poligráficas en Colombia, en particular de la ZCT, por lo que puede contribuir en la aplicación e investigación de la poligrafía en el país, apoyando la labor de los psicólogos jurídicos y forenses que estimen el uso del polígrafo dentro de su peritaje psicológico en casos relacionados con delitos y en otros ámbitos judiciales, ofreciendo 
una perspectiva prácticamente desconocida hasta el momento con respecto al estudio de la exactitud de las técnicas poligráficas en Colombia, ya que propone hacer una evaluación con base en la aplicación de la ZCT en delitos simulados, aunque las generalizaciones que se pueden hacer son limitadas en razón al tamaño de la muestra y por tratarse de una situación preexperimental simulada.

\section{La técnica poligráfica Zona de Comparación Federal (ZCT)}

El examen poligráfico incluye diferentes técnicas, entre ellas Utah, DLST (Directed Lie Screen Test), AFMGQT (Air Force Modified General Question Technique), Bizona, y cada una de ellas cuenta con formatos propios de preguntas y de calificación, por esta razón la Asociación Americana de Poligrafía (APA por sus siglas en inglés) realizó una revisión sistemática de las investigaciones y un meta-análisis de la literatura académica publicada respecto a los criterios de validez de las técnicas poligráficas de la actualidad. Los estudios de validación de las técnicas

poligráficas de la APA se han presentado y aprobado por el Consejo Directivo de este gremio para estandarizar el uso técnicas de valoración en la "Detección Psicofisiológica del Engaño" (PDD sus siglas en inglés) con ciertos niveles de confiabilidad en los criterios de exactitud, ante la crítica que ha generado la publicación de diversos estudios sin criterios unificados de valoración (American Polygraph Association, 2011).

Otro estudio de validación realizado por Senter, Waller y Krapohl (2008; citado por APA 2011) utilizó la técnica poligráfica AFMGQT y obtuvo una decisión no ponderada de exactitud de 0.849 , con una tasa de exámenes inconclusos de 0.015 . Se realizó por medio de la simulación de un atentado con una bomba en una carretera; en el experimento participaron 33 voluntarios que fueron examinados e instruidos para mentir y otras 36 personas examinadas e instruidas para decir la verdad. 
Una de las estrategias más difundidas por su grado de validación en los Estados Unidos es la técnica de Zona de Comparación Federal (ZCT) (American Polygraph Association, 2011). Sus orígenes se registran en la técnica Backster durante la década de los sesentas y es, de hecho, tanto adaptación como desarrollo de dicho método, aunque con el tiempo ambos métodos han desarrollado diferencias sustanciales en características de calificación, reglas de calificación, cortes y número de secuencia de las preguntas del test. La arquitectura básica del test de Backster es la de ubicar una pregunta de comparación excluyente antes de cada pregunta relevante, estrategia que ha sido mantenida en el ZCT (Cullen y Bradley, 2004; Krapohl y Duttin, 2005; citado por Slupski, 2010).

En este punto, es importante aclarar los tipos de preguntas que conforman un "template" o listado de preguntas que se realizan en un examen poligráfico. Durante la entrevista de pretest, todas las preguntas de la ZCT son revisadas con el examinado antes de la toma de registros psicofisiológicos. La siguiente secuencia se utiliza para presentar las preguntas: Relevante de Sacrificio, Relevante, Comparativa, Irrelevante o Neutral y Sintomática.

La pregunta relevante de sacrificio es la primera pregunta del formato ZCT que se refiere al asunto específico y prepara al examinado para la introducción de las preguntas relevantes, es decir, en esta pregunta se menciona el tema concreto por el cual se está realizando el polígrafo. La pregunta relevante evalúa la posible participación directa del evaluado en el asunto. La pregunta comparativa (PLC o PLCQ por sus siglas en inglés) está diseñada para ser una mentira probable para el examinado, la pregunta del PLC debe ser de naturaleza similar pero no relacionada con el crimen o problema específico que se está probando. Estas preguntas deben separarse de la cuestión pertinente en cualquier momento, lugar o categoría. Las preguntas de comparación deben utilizar el mismo verbo de acción, o similar en el verbo acción de naturaleza, 
como el de la pregunta relevante. Las preguntas de comparación deben ser amplias en cuanto al alcance y al tiempo, de manera que puedan captar tantas experiencias anteriores como sea posible. La pregunta irrelevante o neutral es la primera pregunta formulada durante la fase de recolección de datos. Está diseñada para permitir una respuesta orientada que habitúe al evaluado antes de que se haga la pregunta puntuable y se puede usar para que el examinado regrese a la homeostasis cuando ocurra un artefacto en la gráfica. Las preguntas irrelevantes no deben estar relacionadas con el tema que se está evaluando y no se califican. Las preguntas sintomáticas están diseñadas para evaluar un asunto externo que podría ser más significativo para un examinado que los asuntos relevantes y/o de comparación (American Association of Police Polygraphists, 2008).

Los siguientes son algunos ejemplos de preguntas usadas en el protocolo ZCT que pueden ser: neutrales: ¿Las luces están prendidas en este cuarto?, relevantes: ¿robó usted dinero de esa mesa?, de conocimiento: ¿sabe usted con seguridad quién robó ese dinero? (Ansley, 1998).

Para el estudio de las respuestas obtenidas durante el examen de polígrafo, tanto en el pretest como en el test, el análisis cognitivo y lingüístico de las declaraciones suele ser un procedimiento utilizado para discriminar entre declaraciones falsas o verdaderas, así como el análisis de contenido que no siempre resulta suficiente y requiere de un soporte, o al menos de un nuevo tipo de evidencia que permita sustentarlo. Por consiguiente, y pese a los avances científicos en cuanto a la validez interna de las preguntas y la precisión del polígrafo, es necesario que las técnicas utilizadas en los exámenes poligráficos se pongan a prueba en la práctica forense en casos concretos, a manera de pilotos, para definir protocolos y criterios que pueden afrontar las limitaciones de este tipo de evaluación (Arce \& Fariña, 2013). 
Ante el hecho significativo que plantea los avances en la validez y la confiabilidad del polígrafo y de las preguntas utilizadas en el desarrollo de la evaluación psicofisiológica, finalmente se eligió para la presente investigación la técnica poligráfica ZCT: su protocolo es el único que mide complicidad-conocimiento, ya que la inclusión en una versión de la ZCT de una pregunta relevante que establezca conexiones de complicidad ha causado que algunos la consideren como una prueba multifacética. Esto hace que la ZCT se considere más un estudio investigativo que una prueba que busque establecer elementos probatorios. Las preguntas adicionales en la prueba convierten a la ZCT en un cuestionario más rico y útil durante la investigación comparada con un test de asunto único. Sin embargo, la utilidad añadida puede obtenerse a coste de la validez interna, ya que las diferentes coberturas de las preguntas relevantes reducen el enfoque en el número de presentaciones de un asunto único durante la evaluación.

Existe una versión de la técnica poligráfica ZCT denominada IZCT (Integrated Zone Comparison Technique). El desarrollo académico de este test se dio en 1987 a partir del método de Backster (Gordon \& Fleisher, 2012); el formato IZCT posee trece preguntas, dos relevantes, tres flexibles y tres que pueden ser mentiras con criterios de inclusión/exclusión, una pregunta sintomática y cuatro relevantes. La validación del test con varios voluntarios de la Fuerza Aérea Sudafricana mostró un $100 \%$ de precisión de confiabilidad sin incluir resultados inconclusos y $88 \%$ cuando se consideran los resultados inconclusos como errores. No obstante, los autores concluyen que los resultados siempre deben ser matizados respecto a la competencia de los validadores del test como fuente de validez interna en los resultados de las pruebas.

Los resultados de estudios complementarios apoyan la validez de la hipótesis de que las puntuaciones numéricas del método Backster pueden diferenciar el engaño de la verdad con tasas 
que son significativamente mayores que el azar. Los investigadores aducen que los argumentos no son convincentes para diferenciar entre las técnicas ZCT y el método Backster. Ni la lingüística ni el orden de las preguntas técnicas se tienen en cuenta en los protocolos de puntuación numérica. La principal diferencia que podría afectar a las calificaciones numéricas y exactitud entre ambos cuestionarios es el criterio de inserción de preguntas neutrales, es decir, preguntas que no son relevantes cuando el entrevistado suministre datos inexactos en su testimonio o relato de hechos (Nelson, Handler, Adams, \& Backster, 2012).

En este punto es posible observar la viabilidad del núcleo problemático de este estudio, en cuanto a la novedad que implica la utilización del protocolo ZCT y el aporte de esta investigación para la determinación de la exactitud de las técnicas poligráficas en delitos simulados en Bogotá con ambientes experimentales.

\section{Método}

\section{Tipo de estudio}

Esta es una investigación de corte cuantitativo y diseño preexperimental de comparación de grupos con una sola medición, con un grado de control mínimo. Se administró un estímulo a cada grupo según su participación (tomada esta palabra en su sentido más amplio de "estar relacionado con”), y luego se aplicó el polígrafo para determinar la categoría de sus resultados, es decir, NDI, DI o inconcluso INC. 


\section{Tabla 1. Diseño Experimental}

\begin{tabular}{lcc}
\multicolumn{2}{c}{ Diseño Experimental } \\
G1 & $\mathrm{X}$ & 01 \\
$\mathrm{G} 2$ & $\mathrm{X}$ & 02 \\
$\mathrm{G} 3$ & - & 03 \\
\hline
\end{tabular}

La variable independiente de este estudio fueron los tipos de participación en el hurto simulado, cuyos valores son: "responsables", "conocedores" y "grupo control" del experimento. Las variables dependientes fueron los resultados poligráficos, es decir, las medidas psicofisiológicas asociadas al ritmo cardíaco y la conductancia de la piel de las cuales se podrán inferir: "NDI", "DI", e "INC", es decir, los resultados expresados como índices de salida del dispositivo que señalan al sujeto evaluado como aprobado o no en el polígrafo.

Con el objetivo de valorar la relación existente entre el tipo de participación en el hurto (responsable, conocedor y grupo control) y el resultado poligráfico, se formuló la siguiente hipótesis nula:

Ho. No existe relación entre las variables tipo de participación en el hurto y resultado poligráfico.

Como hipótesis de trabajo se formula la siguiente:

H1. Existe relación entre las variables tipo de participación en el hurto y resultado poligráfico.

De la anterior hipótesis, se pueden obtener un conjunto de consideraciones (en adelante indicadas con la letra "C" y su número correspondiente) que plantean una relación significativa 
entre los tipos de participación en el hurto y el resultado poligráfico y en función de las cuales se evaluará la precisión de la técnica ZCT:

C1. Se espera que el grupo 1 "responsables" obtenga como resultado DI.

C2. Se espera que el grupo 2 "conocedores" obtenga en su mayoría como resultado DI, si se consideran como involucrados al ser conocedores-observadores y no delatar.

C3. Se espera que el grupo 3 "grupo control", obtengan como resultado NDI, pues no participaron en el hecho.

\section{Sujetos experimentales}

La muestra estuvo conformada por 30 sujetos experimentales de sexo masculino y femenino, estudiantes de la Universidad Santo Tomás de diferentes facultades. El primer grupo contó con 10 estudiantes, 5 mujeres y 5 hombres (esta distribución sólo fue intencional en este grupo, en los otros grupos no la hay), pertenecientes al grupo de teatro de la Universidad y actores en formación que interpretaron el hurto, cuyo criterio de selección fue su mayor experiencia actoral. El segundo grupo se conformó por 10 estudiantes, nueve hombres y una mujer, grupo denominado "conocedores", que supieron del hecho porque vieron el video del hurto. El tercer grupo, denominado grupo control, contó con 10 sujetos, discriminados en ocho mujeres y dos hombres. Los participantes fueron voluntarios y dieron su consentimiento autorizado para participar en la simulación, ser evaluados en el examen poligráfico y utilizar sus resultados para los fines de la investigación. El rango de edad de la muestra osciló entre los 17 y 31 años. Para el caso del sujeto menor de edad, sus representantes legales autorizaron su participación mediante un consentimiento informado. 


\section{Instrumento}

Se empleó el sistema de polígrafo computarizado LX 4000 marca Lafayette, compuesto por dos bandas "neumo" (variable de respiración), una manga "cardio" (variable de frecuencia cardíaca) y un GSRS (Galvanic Skin Response Sensor). El equipo cuenta además con un sensor de movimiento (en el asiento de la silla) que registra movimientos voluntarios del evaluado y un DAS (Data Acquisition System), que es una caja electrónica que convierte las señales eléctricas en valores digitalizados mediante el software poligráfico.

\section{Procedimiento}

Para efectos del estudio se reunió a todos los sujetos, con excepción del grupo control, con el fin de explicarles el experimento, en razón del cual serían divididos en tres grupos, y posteriormente entrevistados y grabados en video durante el examen poligráfico. En este momento se explicaron las indicaciones éticas y se tomó el consentimiento informado para la realización de la investigación.

La conformación de los grupos correspondió al manejo de la variable independiente: a) simulación del hurto: los actores sustrajeron un computador portátil y un bafle de un recinto universitario; b) observación del video del hurto: tras informarles que se trataba de un hurto simulado con fines experimentales; c) desconocimiento del hurto o grupo control.

El resultado del examen poligráfico se asumió como la variable dependiente, es decir, NDI, DI e INC. Cuando les fue aplicado el examen poligráfico, todos fueron entrevistados con el mismo template, sin ser diferenciados por grupos.

En la siguiente figura se puede observar el template que fue utilizado con todos los sujetos del estudio. 


\begin{tabular}{|c|c|c|c|c|}
\hline$\#$ & 国 ID & Tipo & ?] Texto de Pregunta & $\#$ Respuesta \\
\hline \multicolumn{3}{|c|}{$\exists$ Lista de Preguntas: 1 - ZCTF MAESTRÍA } & \multicolumn{2}{|l|}{ Zona } \\
\hline 1 & 国 & X Comienzo de Gráfica & LA GRÁFICA VAA COMENZAR POR FAVOR PERMANEZCA QUIETO & \\
\hline 2 & 国1 & Irrelevante / Neutral & ESTAMOS EN BOGOTÁ? & $\pm \mathrm{Si}$ \\
\hline 3 & 国SR & Relevante Sacrifico & $\begin{array}{l}\text { CON RELACIÓN AL ROBO DEL COMPUTADOR PORTÁTIL Y EL BAFLE DEL SALÓN } \\
\text { TORREÓN, CONTESTARÁ USTED CON LA VERDAD A MIS PREGUNTAS? }\end{array}$ & $\$ \mathrm{Si}$ \\
\hline 4 & 国01 & Sintomática & TIENE TEMOR DE QUE LE HAGA UNA PREGUNTA QUE NO HAYAMOS REPASADO? & $=$ No \\
\hline 5 & 国1 & $\square$ Control / Comparación & $\begin{array}{l}\text { ANTES DE SUS XX AÑOS, DESEÓ USTED ALGO CON TANTA INTENSIDAD QUE QUISO } \\
\text { ROBARLO? }\end{array}$ & $=$ No \\
\hline 6 & 国R1 & $\square$ Relevante & $\begin{array}{l}\text { ROBÓ USTED EL COMPUTADOR PORTÁTIL Y EL BAFLE REPORTADOS PERDIDOS DEL } \\
\text { SALÓN TORREÓN? }\end{array}$ & $=$ No \\
\hline 7 & 国 2 & $\square$ Control / Comparación & $\begin{array}{l}\text { ANTES DE INGRESAR AL XX SEMESTRE DE LA UNIVERSIDAD, LE ROBÓ USTED ALGO A } \\
\text { ALGUIEN? }\end{array}$ & $=$ No \\
\hline 8 & 国R2 & $\square$ Relevante & $\begin{array}{l}\text { USTED ROBÓ EL COMPUTADOR PORTÁTIL Y EL BAFLE REPORTADOS PERDIDOS DEL } \\
\text { SALÓN TORREÓN? }\end{array}$ & $=$ No \\
\hline 9 & 国O2 & Sintomática & ESTÁ USTED SEGURO QUE SÓLO LE HARÉ LAS PREGUNTAS QUE REPASAMOS? & $\# \mathrm{Si}$ \\
\hline 10 & 国c3 & $\square$ Control / Comparación & ENTRE SUS XX Y SUS XX AÑOS, COMETIÓ USTED UN ACTO DESHONESTO? & $=$ No \\
\hline 11 & 国R3 & Relevante & $\begin{array}{l}\text { PARTICIPÓ USTED INTENCIONALMENTE EN EL ROBO DEL COMPUTADOR PORTÁTIL Y EL } \\
\text { BAFLE REPORTADOS PERDIDOS DEL SALÓN TORREÓN? }\end{array}$ & $=$ No \\
\hline 12 & 国xx & $\times \propto$ Fin de Gráfica & $\begin{array}{l}\text { LA GRÁFICA HA TERMINADO, POR FAVOR PERMANEZCA QUIETO HASTA QUE SE LE } \\
\text { INDIQUE. }\end{array}$ & \\
\hline 13 & & & & \\
\hline
\end{tabular}

Figura $N^{\circ}$ 1. Template.

Siguiendo el protocolo internacional del examen poligráfico se grabó en video cada uno de los exámenes y se les entrevistó, solicitando nuevamente su consentimiento en esta ocasión para la realización del examen poligráfico y se explicó el motivo del mismo, esto es, la comisión del hurto y el grado de conocimiento acerca del mismo; se les explicó el instrumento psicofisiológico y sus componentes, así como aspectos básico del SN y en particular del SNA en cuanto a las reacciones que se generan ante el temor de ser descubierto; se entrevistó por una hora sobre aspectos de su historia personal; se repasaron todas las preguntas; finalmente se ubicaron los sensores y se inició el registro de las reacciones psicofisiológicas ante las preguntas del template. El poligrafista seguidamente calificó las gráficas e identificó el resultado, si era NDI o DI culminaba la evaluación, y si era INC invitaba a la repetición del examen. La ZCT requiere mínimo de tres gráficas y máximo de cinco. El sistema de calificación que se utilizó fue el ESS o Empirical Scoring System, que consiste en asignar un valor positivo o negativo a la reacción más grande, dependiendo en qué tipo de pregunta se presente la mayor reacción. El 
valor positivo $(+1$ o +2$)$ se asignará si la reacción más grande se presentó ante una pregunta comparativa; el puntaje negativo $(-1$ o -2$)$ si la reacción se presentó a la pregunta relevante. Se asigna un puntaje de cero (0) si las reacciones a ambos tipos de preguntas son muy similares.

Para efectos de la investigación se pidió a los participantes que respondieran a las preguntas del polígrafo como si se enfrentaran a una situación de la vida real. Se elaboraron tablas de frecuencia descriptivas por cada variable y discriminadas por grupo y sexo. Mediante la comparación de los resultados obtenidos en los exámenes entre los distintos grupos participantes, se podrá dar respuesta al núcleo problemático.

Para determinar la exactitud de detección de engaño de la técnica ZCT, es decir, establecer que el grado de credibilidad de los participantes no se debió a un efecto aleatorio sino a la pertinencia del instrumento y la técnica empleados, se aplicó una prueba Chi-Cuadrado a la tabla de contingencia resultado de los datos observados. El análisis de los datos obtenidos por el polígrafo se hizo por medio del programa estadístico SPSS.

Todo el estudio se realizó bajo el contexto de buenas prácticas éticas que propenden por el bienestar humano y la dignidad de las personas que participaron, con pleno conocimiento de las normas legales y de los estándares profesionales que regulan la conducta de la investigación con participantes humanos (Código Deontológico \& Bioético de Psicología, 2006).

También se tuvieron en cuenta los principios éticos de los psicólogos y del código de conducta de la American Psychological Association (APA) con sus enmiendas del año 2010, las cuales advierten sobre el riesgo de daño a participantes que eventualmente puede surgir en las investigaciones, para establecer que la situación experimental no es nociva para ninguno de ellos.

Respecto a las grabaciones del hurto simulado, se obtuvo el permiso de los funcionarios a cargo de las instalaciones y se hizo uso del consentimiento informado para cada uno de los 30 
sujetos experimentales de la investigación. Además, los psicólogos ofrecieron a los participantes la oportunidad de obtener información sobre los objetivos del método poligráfico en esta investigación y tomarse las medidas razonables para corregir cualquier malentendido que pudiera producirse.

\section{Resultados}

La distribución de los resultados obtenidos durante el desarrollo de la investigación es la siguiente:

15 participantes del total obtuvieron NDI ante el polígrafo; 11 obtuvieron DI; y sólo 4 obtuvieron un resultado Inconcluso, ya que sus reacciones psicofisiológicas no permitieron emitir un diagnóstico definitivo, NDI o DI, pese a ser verificadas por un poligrafista Quality Control. No obstante, cuando se detalla cada grupo se observa que, en el primer grupo, 8 de los 10 sujetos obtuvieron como resultado DI, lo que vendría a confirmar la primera consideración (C1), según la cual en este grupo sería predominante el resultado DI, mientras que los dos sujetos restantes (2 de 10) resultaron inconclusos.

En el segundo grupo, a quienes les fue presentado el video del hurto simulado, 7 de 10 sujetos obtuvieron NDI ante el polígrafo; mientras que los otros tres sujetos para este caso, presentaron DI como resultado poligráfico.

En el tercer grupo o grupo control, la mayoría (8 de 10 participantes) superó las preguntas de la ZCT con NDI; sin embargo, no fue posible emitir un diagnóstico concluyente de los registros de dos sujetos (un hombre y una mujer), por lo que su resultado fue INC a pesar de su inocencia dentro del experimento.

Adicionalmente se realizó un análisis con la prueba de Chi-Cuadrado, el cual tiene como principal objetivo comprobar la existencia o inexistencia de relaciones de dependencia entre dos 
variables categóricas cuyo nivel puede ser ordinal o nominal; siendo éste último el que corresponde a la presente investigación, permite confirmar una de la hipótesis, la nula (Ho) o la de trabajo (H1). El análisis de Chi-Cuadrado se realizó con el paquete estadístico SPSS, y arrojó los siguientes resultados:

Tabla 2. Resultados Prueba de Chi- Cuadrado

\begin{tabular}{lccc}
\hline & \multicolumn{3}{c}{ Resultado Chi- Cuadrado } \\
\cline { 2 - 4 } & Valor & Gl & $\begin{array}{c}\text { Significación } \\
\text { asintótica }\end{array}$ \\
\hline Chi- Cuadrado & 18,509 & 4 & 0.0010 \\
\hline $\begin{array}{l}\mathrm{N}^{\circ} \text { de casos } \\
\text { válidos }\end{array}$ & 30 & 7 & 0 \\
\hline
\end{tabular}

La hipótesis nula (Ho), según la cual no existe relación entre las variables tipo de participación en el hurto y resultado poligráfico, debe rechazarse si la significación asintótica (probabilidad de que el estadístico que se está probando muestre un valor igual o inferior que el nivel de significación deseado) muestra un valor de Chi-Cuadrado inferior a 0,05. Al analizar en SPSS el estadístico descriptivo de Chi-Cuadrado el resultado obtenido fue un coeficiente de 0,0010, razón por la cual se descarta esta hipótesis, manteniendo la hipótesis de trabajo (H1), que confirma la relación de las variables analizadas.

\section{Discusión}

Los resultados evidencian dispersión de la muestra y discriminación a través del uso del instrumento psicofisiológico. Estos resultados permiten confirmar la primera consideración (C1), según la cual el grupo 1 obtendría el resultado DI predominantemente.

El segundo grupo presentó tres sujetos con resultado DI; esto se puede explicar, presumiblemente, porque estos participantes, sin ser responsables del hurto, experimentaron emociones morales como culpa o vergüenza, porque aunque informaron del hecho durante la 
entrevista no pudieron identificar a los responsables y probablemente se autoevaluaron como cómplices por el hecho de no denunciar. Esta inferencia está soportada en las entrevistas hechas a los sujetos en el momento en que se les preguntó sobre el hurto y qué consecuencias deberían tener quienes lo cometieron.

En este caso se descarta la segunda consideración (C2), según la cual se esperaba que en su mayoría el grupo 2 mostrara indicación de engaño (DI) por su conocimiento del hurto; los resultados de la prueba mostraron lo contrario a lo esperado para $\mathrm{C} 2$, es decir, la mayoría presentó NDI, por lo cual no se debe equiparar la observación del delito con la participación en el mismo. Por esta razón 7 de 10 de los voluntarios obtuvieron NDI en la evaluación de la credibilidad de sus testimonios por medio de la ZCT.

En el tercer grupo, la mayoría superó las preguntas de la ZCT con el resultado NDI. Estos hallazgos son coherentes con lo esperado en la tercera consideración (C3), ya que la mayoría del grupo 3 presentó NDI en la prueba de polígrafo. Ante estas situaciones el procedimiento poligráfico habitual conmina a repetir el examen, lo cual habría permitido clarificar la situación, pero estos participantes no lo permitieron, bajo el argumento de no contar con el tiempo suficiente para la realización del mismo en un segundo momento.

Al analizar los resultados anteriores con la prueba estadística Chi-Cuadrado, el resultado obtenido fue un coeficiente de 0,0010. Dicho valor significa que la investigación muestra una evidencia estadísticamente significativa que permite aceptar la hipótesis de trabajo, lo que quiere decir que el resultado del examen poligráfico sí se relaciona con la variable independiente.

De los 30 exámenes poligráficos realizados, se observó una precisión en la detección de 23 casos, lo que equivale al $76 \%$, es decir, el $24 \%$ restante presentaron respuesta inconclusas o inexactas, sin embargo, este índice no resulta suficiente para alcanzar los estándares mínimos 
requeridos por la American Polygraph Association que requiere de un 80\% (APA, 2011); de modo que la exactitud de la técnica poligráfica ZCT identificada en este estudio es levemente inferior a lo exigido, aunque se considera posible haber logrado una precisión más elevada si no hubieran habido resultados inconclusos, ya sea por la repetición de los exámenes poligráficos o al descartar a esos participantes e incluir otros en la investigación que alcanzaran resultados DI o NDI.

Con relación al presente estudio y su procedimiento, investigaciones como las de Bradley, Malik, \& Cullen (2011), refieren la utilización de crímenes simulados en ambientes experimentales con el fin de determinar factores como la memoria a la hora de obtener resultados poligráficos, usando grupos que cometían directamente el delito y grupos control, concluyendo que las tasas de detección y el elemento recordado en la situación, fueron mayores para el grupo de laboratorio, a quienes se les habían explicitado de forma consciente todos los ítems, que para el grupo control.

De acuerdo con el estudio arriba citado, una investigación posterior de Gamer (2012) acerca del artículo realizado por los anteriores autores, indica que los crímenes simulados, pero más naturales, pueden llegar a arrojar múltiples medidas de respuestas fisiológicas y técnicas de medida y clasificación mejoradas. Para este estudio, en apoyo con los resultados del antes citado, el hurto simulado no contó con exceso de detalles en su realización, excepto las indicaciones que se les dieron a los actores, esto es, asumir en su totalidad el papel, el rol de responsables del hurto.

Finalmente, otro estudio que se realizó mediante el uso de crímenes simulados efectuado por Visu-Petra, Buş \& Miclea (2011), y con el que se pretendía determinar la exactitud de detección de la técnica poligráfica CIT (Test de Información Oculta) vs el CIT basado en RT 
(Tiempos de Reacción) en un crimen simulado, utilizando medidas psicofisiológicas de las respuestas a las preguntas de la prueba de polígrafo y los tiempos de reacción, permitió confirmar la identificación de los efectos de la información oculta utilizando las dos técnicas de evaluación y reveló que los dos métodos tienen rangos exactitud similar para la detección de la mentira.

Estos estudios permiten resaltar la importancia que tiene la utilización de situaciones simuladas, en particular de delitos, a la hora de determinar la exactitud de una técnica poligráfica, cualquiera que esta sea. El concepto de exactitud a que nos referimos en este estudio es el que define la Asociación Americana de Poligrafía (2011) como el grado en que el resultado de una prueba corresponde con el propósito para el cual el examen fue diseñado. En el campo de los exámenes PDD, el criterio de exactitud denota la habilidad de una combinación de técnicas de evaluación y calificación, para discriminar entre examinados honestos y deshonestos. El criterio de exactitud es una forma de validez y en algunos reportes de investigación puede ser referido como exactitud en la decisión o, simplemente, como exactitud.

Como dato cualitativo se encontró que en los relatos de grupo 3, los evaluados aportaron datos imaginarios y varias situaciones a pesar de desconocer el hecho, incurriendo en lo que se denomina en psicología del testimonio como confabulación. La definición operativa que proponen Lorente Rovira, E., Berrios, G., McKenna, P., Moro Ipola, M., \& Villagrán Moreno, J. M. (2011), señala que existen cuatro aspectos característicos de las confabulaciones: a) Son falsos recuerdos en el contexto de la recuperación que, a menudo, contienen detalles falsos dentro de su propio contexto también; por tanto, algunas confabulaciones pueden ser recuerdos reales mal situados en el tiempo, mientras que otras parecen carecer de cualquier base real; b) La persona no es consciente de que está confabulando y a menudo ni siquiera es consciente de la 
existencia de un déficit de memoria. Por tanto, las confabulaciones no se producen de manera intencional y probablemente no son el resultado de mecanismos compensatorios; c). Las personas pueden actuar de forma consecuente con sus confabulaciones, reflejando su creencia genuina en el falso recuerdo; d). Son más evidentes cuando se pide una recolección autobiográfica. Sin embargo, existe evidencia de casos de confabulaciones espontáneas que no estaban directamente relacionadas con la vida de los pacientes.

Es importante destacar que se logró determinar la exactitud de la técnica ZCT en 23 casos, mientras que en los 7 restantes (24\%) no se pudo determinar la credibilidad del testimonio, ya que se obtuvieron resultados inconclusos; esta situación se debió solventar con la repetición del examen, pero los investigadores dependían de la disponibilidad de los participantes. Para futuras investigaciones se sugiere descartar estos sujetos experimentales por otros que permitan la evaluación completa.

\section{Conclusiones}

La técnica ZCT alcanzó una exactitud del 76\%, dado que mostró resultados acertados en 23 de los 30 casos expuestos al análisis, lo que es un indicador aceptable para la detección del engaño en esta investigación, particularmente respecto a los grupos 1 y 3, pues arrojaron resultados admisibles respecto a la precisión de la evaluación de la credibilidad, por lo cual se puede considerar a la poligrafía como una herramienta que coadyuva en la evaluación de la credibilidad del testimonio en la psicología forense. Los resultados de exactitud en esta investigación resultan similares con otras investigaciones previas; no obstante, los resultados no se pueden generalizar en razón a que la muestra de este experimento no fue representativa.

Los resultados del grupo 2 presentaron una particularidad: el conocimiento del hecho actuó como variable interviniente en el grado de acierto de la ZCT ya que, aunque todos los 
miembros de este grupo siguieron las instrucciones, unos fueron clasificados como NDI y otros DI, de modo que el conocimiento sí afectó los resultados esperados, pues finalmente este grupo confirmó que no es necesario saber del hecho para sentirse cómplice de un delito, evidenciado por la mayoría de resultados NDI que obtuvo el grupo.

Las cualificaciones para los examinadores de polígrafo con la técnica ZCT deben retroalimentarse de investigaciones como esta, ya que estos hallazgos evidencian que la valoración del examinador es un factor importante que podría evitar los sesgos a la hora de realizar la indagación con los evaluados, de la misma manera que permite mejorar la precisión en la aplicación de las técnicas de polígrafo.

Con esto se puede afirmar que la técnica poligráfica Zona de Comparación Federal resulta una herramienta con un grado admisible de exactitud como instrumento inicial del análisis de credibilidad del testimonio en temas o hechos específicos, lo cual beneficiaría la labor de los psicólogos forenses en casos relacionados con delitos. Sin embargo, es importante señalar que se requieren más estudios de aplicación para determinar la exactitud de las diferentes técnicas poligráficas con el fin de lograr un mayor grado de legitimidad ante la comunidad académica especializada y puedan llegar a ser consideradas como una prueba dentro del proceso penal colombiano y como un elemento de uso consuetudinario por parte de los psicólogos jurídicos y forenses para la elaboración de sus peritajes. Por lo tanto, los programas académicos afines como la psicología y el derecho deberían invertir esfuerzos en aplicaciones investigativas con la ZCT para que en un futuro se emplee con frecuencia en los casos que sea requerida y no como un elemento aislado en la investigación científica.

Para próximos estudios se sugiere contar con muestras más amplias, así como reemplazar a los sujetos experimentales que obtengan en el polígrafo resultados inconclusos o asegurar su 
participación hasta que sea posible reexaminarlos y emitir un diagnóstico poligráfico claro. Se considera que si estas limitaciones hubieran podido superarse en el presente estudio previendo estos factores de tipo logístico, los niveles de exactitud de la técnica ZCT serían más elevados.

\section{Referencias}

American Association of Police Polygraphists (2008). Examiner Handbook, pp. 13- 20.

American Polygraph Association (2011). Estudio Meta Analítico del Criterio de Exactitud de las Técnicas Poligráficas Validadas. Polygraph, 40(4), 194-305. Recuperado de http://www.polygraph.org

Ansley, Norman (1998). The Zone Comparison Test. Polygraph, 27(2), pp. 108-122.

Arce, R., y Fariña, F. (2013). Psicología forense experimental. Testigos y testimonio. Evaluación cognitiva de la veracidad de testimonios y declaraciones. En Delgado, S. (Dir. Tratado), y Delgado, S. y. Maza, J. (Coords. Vol.), Tratado de medicina legal y ciencias forenses (Vol. V. Psiquiatría legal y forense, pp. 21-46). Barcelona: Bosch.

Ayoub, A. y Ashraf, M. (2016). Forensic Polygraph in Crime Investigation: A Case Study. Arab Journal of Forensic Sciences and Forensic Medicine, 1(3), pp. 340-345.

Barret K. y Barman S. (2013). Ganong Fisiología Médica24a Edición. México: McGraw Hill.

Bohada, D., García, E., Ortíz, J. y Oses, M. (2011). Uso de la poligrafía en el Instituto Latinoamericano de Poligrafía (LPI) desde la psicología jurídica- forense. Universidad Santo Tomás. 
Bradley, M., Malik, F., \& Cullen, M,. (2011). Memory Instructions, Vocalization, Mock Crimes, and Concealed Information Tests With a Polygraph. Perceptual and Motor Skills, 113 (3), $840-858$

Budahazi, A. (2012). Conditions and Requirements of Polygraph Examination. European Polygraph, 6,3(21), pp. 161-180.

Corte Suprema de Justicia, Sala de Casación Penal, Proceso N²647. Decisión de 01 de agosto de 2008 .

Código de Procedimiento Penal Colombiano. (2004). Bogotá, Colombia

de Psicólogos, C. C. (2012). Deontología y Bioética del ejercicio de la Psicología en Colombia. Colegio Colombiano de Psicólogos.

Espinosa, A. (2011). La Psicología del Testimonio. (Ed), Psicología Jurídica Iberoamericana (197-229) Bogota: Manual Moderno

Gamer, M. (2012). Validity of the Concealed Information Test in Realistic Mock Crime Scenarios: Comment on Bradley, Malik and Cullen. Perceptual and Motor Skills, 115(2), pp. 427-431.Golaszewski, Marcin (2012). Validated Techniques and scoring models PDD Test Data Analysis. European Polygraph, 6(22). Recuperado de: http://www.polygraph.pl/vol/EP2012-4-Golaszewski.pdf

Gómez, C. y Farfán, F. (2014). El polígrafo y su utilización como acto de investigación en el derecho sancionatorio. Revista Derecho Penal y Criminología, 35(98), pp. 131-179.

Gómez, C., Farfán, F. y López R. (2016). El polígrafo como mecanismo de investigación en el proceso penal. Discusiones, naturaleza y viabilidad. Bogotá. Defensoría del Pueblo. 
Gordon, N. y Fleisher, W. (2012). Effectiveness of Integrated Zone Comparison Technique (IZCT) with Various Scoring Systems in a Mock Crime Experiment in Students. European Polygraph, 6, 1(19), pp. 5-17.

Guerrero, Y. (2013). Del instrumento de investigación polígrafo "examen psicofisiológico”: un análisis sobre su valor probatorio en el ámbito penal en Colombia. Recuperado de http://repository.ucatolica.edu.co:8080/xmlui/handle/10983/2596.

Lessons from Polygraph-based Approaches. En Frontiers of Psychology, 4 (14). Recuperado de: 10.3389/fpsyg.2013.00014

Lorente Rovira, E., Berrios, G., McKenna, P., Moro Ipola, M., \& Villagrán Moreno, J. M. (2011). Confabulaciones (I): Concepto, clasificación y neuropatología.

Manzanero, A.L. (2010). Hitos de la historia de la psicología del testimonio en la escena internacional. Boletín de Psicología, (100), pp. 89-104.

Manzanero, A.L. y Muñoz, J.M. (2011). La prueba pericial psicológica sobre la credibilidad del testimonio: Reflexiones psico-legales. Madrid: SEPIN. Recuperado de: http://eprints.ucm.es/12544/1/CREDIBILIDAD_DEL_TESTIMONIO.pdf

Nelson, R., Handler, M., Adams, G. y Backster, C. (2012) Survey of Reliability and Criterion Validity of Backster Numerical Scores of You-Phase Exams from Confirmed Field Investigations. Polygraph, 41(2), pp. 127-135.

Quejereta, Luis M. (1999) Validez y credibilidad del testimonio. La psicología forense experimental. En EGUZKILORE, $\quad \mathrm{N}^{\mathrm{o}}$ 13, 157-168. Recuperado de: 
http://www.ehu.eus/documents/1736829/3343253/Eguzkilore\%2B13-

12.\%2BQuerejeta.pdf

Ruiz, J., Llor, E. y Valverde, M. (2013). Valoración de la Credibilidad del Testimonio: Aplicación del Modelo Reality Monitoring. En Revista Internacional de Psicología, 12 (2), pp. 1-29.

Slupski, Ch. (2010). Técnica de Comparación Zona Federal. Instituto Americano Internacional de Poligrafía: Estados Unidos. Recuperado de https://polygraphschool.com.

Superintendencia de Vigilancia y Seguridad Privada. Resolución 2593de 2003.

Tapias, A. (2012) Introducción judicial del polígrafo a través de la pericia psicológica forense a presuntos agresores sexuales. Rev. Crim., Volumen 54, número 1, p. $405-417$ enero-junio 2012, Bogotá, D. C., Colombia

Tapias A., Avendaño A., Fuentes A y Zaldúa J. (2003). Viabilidad de la aplicación en Colombia de las técnicas psicológicas: el polígrafo, el análisis de estrés de voz, el análisis de contenido basado en criterios, la hipnosis y la entrevista asistida con drogas. Recuperado de htpp://psicologiajuridica.org/psj97.html.

Vilariño, M., Formosinho, M., y Jesús, P. (2012). Obtención del testimonio y evaluación de la credibilidad. En International Journal of Developmental and Educational Psychology, 1 (1), vol. 1, pp. 599-608 (ISSN: 0214-9877).

Visu-Petra, G., Bus, I., \& Miclea, M. (2011). Detecting concealed information from a mock crime scenario by using psychophysiological and RT-based measures. Cognitive, Creier, Comportament, 15(1), 19-37 
Zhang, X. (2011). The Evolution of Polygraph Testing in the People's Republic of China. En Polygraph, 40 (3), pp. 181-193. 